\title{
ATRAÇÃO DE MIRACÍDIOS DE SCHISTOSOMA MANSONI POR HOSPEDEIROS INVERTEBRADOS COMPORTAMENTO DE MIRACDIOS FRENTE A GIRINOS DE HYLA FUSCOVARLA
}

\author{
Beatriz de C. Brasio* \\ Luiz Augusto Magalhães** \\ Joseph Miller*** \\ José F. de Carvalho****
}

\begin{abstract}
BRASIO, B. de C. et al. Atração de miracídios de Schistosoma mansoni por hospedeiros invertebrados. Comportamento de miracídios frente a girinos de Hyla fuscovaria. Rev. Saúde públ. S. Paulo, 19: 18-27, 1985.
\end{abstract}

RESUMO: Analisou-se a atração de miracídios de Schistosoma mansoni das linhagens BH e SJ frente a seus vetores simpátricos e alopátricos tendo-se em consideração a possibilidade da presença de substâncias quimiotáxicas emanadas dos moluscos. Estudou-se também o comportamento desses miracídios frente a girinos de Hyla fuscovaria. Foi verificado que houve atração dos miracídios pelos moluscos vetores e que essa atração foi mais evidente quando a larva era colocada frente ao seu hospedeiro simpátrico. As observações realizadas no decorrer da experiência demonstraram a presença de substâncias miraxonais emanadas pelos moluscos.

UNITERMOS: Schistosoma mansoni Biomphalaria glabrata. Biomphalaria tenagophila. Hyla fuscovaria.

Kloetzel $^{6,7}$ (1958, 1960), observando o comportamento de miracídios de Schistosoma mansoni em presença do molusco hospedeiro Biomphalaria glabrata, notou que "nas proximidades do caramujo os miracídios, que antes se movimentavam em linha reta e a grande velocidade, diminuem a velocidade, às vezes estancam, curvam o corpo e rodopiam, como em busca de algo que pressen. tem e ficam pelas imediações; além disso, o rastro deixado pelo molusco no fundo do vidro é igualmente objeto de curiosidade". Concluiu, aquele autor, pela existência de quimiotropismo, salientando que sua ação se faz somente nas proximidades do molusco.

Barbosa ${ }^{1,2,3}(1960,1965)$ contestou a existência do quimiotropismo, acentuando que a extraordinária atividade do miracídio o leva aleatoriamente ao caramujo e que seu acentuado tigmotropismo faz com que a larva penetre indiferentemente em planorbideos, fisídeos, ampularídeos e até em larvas de batráqueos.

Usando um aparelho de quatro câmaras, Etge- e Decker ${ }^{4}$ (1963) observaram que os miracídios de $S$. mansoni eram atraídos moderadamente pelos moluscos vetores $B$. $g l a$ brata, mas não por moluscos Helisoma anceps e Bulinus sp, verificando que a quimiotaxia observada apresentava certo grau de especificidade.

Estudos feitos por Upatham ${ }^{17}$ (1972), em habitats simulados de planorbídeos, mostraram que miracídios de $S$ mansoni, quando introduzidos nestes habitats, concentravam.

- Bolsista da FAPESP, junto ao Departamento de Parasitologia do Instituto de Biologia da Universidade Estadual de Campinas (UNICAMP) - Caixa Postal 6109 - 13100 - Campinas, SP - Brasil.

* Do Departamento de Parasitologia do Instituto de Biologia da Universidade Estadual de Campinas (UNICAMP) - 13100 - Campinas, SP - Brasil.

*** Do Instituto de Química da Universidade Estadual de Campinas (UNICAMP) - 13100 - Campinas, SP - Brasil.

**** Do Instituto de Matemática, Estatística e Clências da Computação da Universidade Estadual de Campinas (UNICAMP) - 13100 - Campinas, SP - Brasil. 
BRASIO, B. de C. et al. Atração de miracídios de Schistosoma mansoni por hospedeiros invertebrados. Comportamento de miracidios frente a girinos de Hyla fuscovaria. Rev. Saride públ., S. Paulo, 19:1827,1985 .

-se rapidamente nas margens, devido ao fato destas larvas apresentarem fototropismo positivo e geotropismo negativo. Os caramujos, geralmente, são encontrados nas margens de lagos e rios, fixados em plantas ou pedras, - que facilita a aproximação das larvas; a atração química deverá ocorrer então quando os miracídios estiverem muito próximos ao caramujo.

O processo de estimulação do miracídio foi descrito por Manson e Fipp ${ }^{12}$ (1977) e Roberts e col. ${ }^{16}$ (1980) como contendo duas etapas: a primeira constando de um contacto inicial dentro do "espaço do caramujo" (Wil. $\left.\operatorname{son}^{18}, 1970\right)$, conduzindo a um aumento da velocidade angular (taxa de volteios) do miracídio quando este penetra numa região onde há um decréscimo abrupto dos componentes da SCW*. $O$ efeito principal desta mudança de comportamento é a retenção do miracídio em áreas de alta concentração do estimulante. Este fenômeno poderia ser provocado por pequenas moléculas ou fons inor. gânicos e também por peptídeos (Manson e Fipp $\left.^{12}, 1977\right)$.

$\mathrm{Na}$ segunda etapa, após o contacto inicial do miracídio com "o espaço ativo do molusco", outras substâncias como aminoácidos e ácidos graxos estimulariam a larva a retor. nar e manter contacto com o tegumento do caramujo, ocorrendo então a penetração (Manso e Fipp ${ }^{12}$, 1977).

0 presente estudo visou verificar a atração de miracídios por seus hospedeiros inver. tebrados e a possibilidade da ocorrência de especialidade no desempenho dos mecanismos de atração dos vetores das linhagens de $S$. mansoni oriundas de Belo Horizonte, MG (linhagem BH) e do Vale do Rio Paraíba do Sul, SP (linhagem SJ). Paraense e Corrêa ${ }^{13}$ 14, 15 (1963), ressaltaram que estas duas linhagens estão vinculadas a hospedejros intermediários de espécies diferentes, $B$. glabrata e $B$. tenagophila, respectivamente, havendo resistência à infecção cruzada. E observada competição entre estas duas espécies de planorbídeos (Magalhães ${ }^{8}, 1966$; Kawazoe e col. $\left.{ }^{5}, 1980\right)$.

\footnotetext{
* Snail conditioned water.
}

Utilizamos ainda girinos de Hyla fuscovaria para verificar uma possivel atração de larvas de batráquio sobre miracídios de $S$. mansoni.

\section{MATERIAL E MÉTODOS}

No decurso da experiência utilizamos miracídios de $S$. mansoni das linhagens $\mathrm{BH}$ (Belo Horizonte, MG) e SJ (São José dos Campos, Vale do Rio Paraíba do Sul, SP). Os esquistossomos dessas duas linhagens são mantidos na natureza, respectivamente, em $B$. glabrata e $B$. tenagophila.

No laboratório estas linhagens foram mantidas utilizando-se moluscos simpátricose como hospedeiro vertebrado camundongos albinos.

Os miracídios $\mathrm{BH}$ e $\mathrm{SJ}$ foram obtidos a partir de ovos coletados nas fezes de camundongos com cerca de 40 dias de infecção.

Para a verificação do comportamento das larvas frente aos moluscos utilizamos um aparelho de vidro composto de duas câmaras circulares com $30 \mathrm{~mm}$ de diâmetro unidas por um canal de $40 \mathrm{~mm}$ de comprimento por $11 \mathrm{~mm}$ de largura (Fig.).

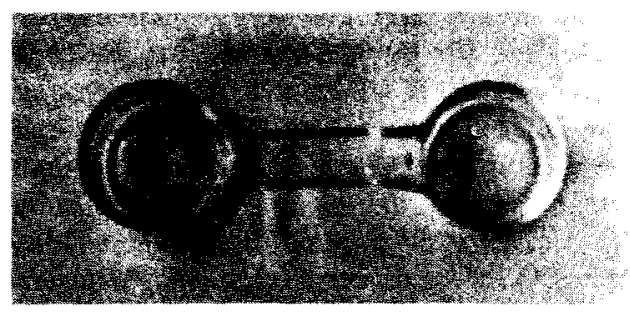

Figura - Aparelho de vidro, composto de duas câmaras, utilizado nos experimentos.

Para a realização dos experimentos, o aparelho contendo água recebia um exemplar de B. tenagophila ou ainda um girino de $\mathrm{Hyla}$ fuscovaria, aleatoriamente colocados em uma das câmaras. Os moluscos planorbídeos utilizados mediam cerca de $10 \mathrm{~mm}$ de diâmetro máximo.

Dez a quinze miracídios eram colocados no centro do canal com uma seringa e observados com auxílio de lupa estereoscópica. 
BRASIO, B. de C. et al. Atraçăo de miracidios de Schistosoma mansont por hospedeiros invertebrados. Comportamento de miracidios frente a girinos de Hyla fuscovaria. Rev. Saide públ., S. Paulo, 19:1827,1985 .

A movimentação das larvas era observada durante cerca de $15 \mathrm{~min}$. Anotávamos o comportamento dos miracídios frente aos caramujos ou girinos.

Foram realizados experimentos colocando-se miracídios BH ou SJ frente a $B$. glabrata (na ausência e na presença de luz), $B$. tenagophila (na ausência e na presença de luz) e Hyla fuscovaria (na presença de luz).

Quando realizamos os experimentos na presença de luz, cuidamos para que a intensidade luminosa nas duas câmaras fosse a mesma.

Os experimentos realizados na "ausência de luz" foram feitos com focos de luz desligados, porém sob discreta luminosidade ambiente (penumbra).

Para cada um dos tipos de experimentos fizemos um mínimo de vinte observaçoes procurando, em todos eles, manter as mesmas condiçס̃es de luminosidade e o mesmo número de miracídios.

Após a realização de cada observação, 0 aparelho era lavado, cuidadosamente, para evitar que vestigios de muco e fezes pudessem interferir em outras observaçðes.

0 aparelho de duas câmaras também foi usado para observar o comportamento de miracídios BH ou SJ colocados na presença simultânea de $B$. glabrata e $B$. tenagophila. Para isso colocávamos, com o aparelho cheio de água, um exemplar de $B$. glabrata em uma câmara e um exemplar de $B$. tenagophila na outra càmara. Os miracídios eram colocados na regiăo média do canal e observados durante $15 \mathrm{~min}$ com lupa estereoscópica e em presença da luz. Após decorrido este tempo, contávamos o número de miracídios que migrava para cada câmara. Este procedimento foi repetido sessenta e uma vezes, utilizando. -se sempre novos espécimes de moluscos.

Antes de nos decidirmos pela utilização do aparelho de duas câmaras, testamos um aparelho em forma de " $Y$ " contendo duas câmaras nas extremidades da bifurcaçăo. Neste caso os miracídios eram colocados na extremidade distal do duto mediano do $Y$.

Testamos também um aparelho constituido por uma câmara central ligada por canaletas a quatro outras câmaras. Este aparelho tinha forma de cruz. Neste caso os miracidios eram colocados na câmara central.

\section{RESULTADOS}

Na Tabela 1 estão apresentados os resultados finais desta experiência.

Resultaram deste experimento várias observaçбes que acreditamos merecer serem ressaltadas. Observamos que quando utilizamos o aparelho com uma das câmaras contendo somente água, miracídios que para ela se dirigiram nadavam durante algum tempo no vestíbulo da câmara, sem direção definida; muitos retornavam ao canal e dirigiam-se à câmara onde havia um caramujo. Os miracídios que inicialmente entraram na câmara, que continha o caramujo, raríssimas vezes retornavam ao canal.

A presença do muco liberado pelos cara. mujos no vestíbulo das câmaras exerceu forte poder de atração sobre os miracídios. Assim que percebiam a existência do muco, os miracídios passavam imediatamente a rodopiar ao redor dessa secreção, ou entáo tentavam penetrá-la; nem mesmo a presença de um caramujo a pequena distância era suficiente para afastá-los do muco.

As fezes de caramujos tambem exerceram atração sobre os miracídios, se bem que por pequeno período de tempo, após o qual as larvas passavam a reagir à presença das fezes como a qualquer outro objeto estranho colocado no aparelho, e então dirigiam-se ao caramujo ou ao seu muco.

Quando fraturávamos a concha do molusco provocando a liberação de hemolinfa, observamos que os miracídios preferiam permanecer nadando ao redor da concha ou tentavam penetrá-la. Neste caso, o número de miracídios que se concentrava ao redor do tegumento dos moluscos foi sempre menor que o número dos que se colocavam ao redor da concha fraturada.

Os miracídios quando se aproximavam dos caramujos simpátricos permaneciam nadando e rodopiando por mais tempo ao redor do molusco, talvez devido à ação especifica de substâncias miraxonais emanadas por eles. Quando em presença de cara. 
BRASIO, B. de C. et al. Atraçđo de miracídios de Schistosome mansoni por hospedeiros invertebrados. Comportamento de miracidios frente a girinos de Hyla fuscovaria. Rev. Saíde pribl., S. Paulo, 19:18. 27, 1985.

T A B E L A 1

Média numérica e coeficiente de variaçto da percentagem de miracídios que entraram na câmara contendo moluscos ou girinos (câmara A).

\begin{tabular}{|c|c|c|c|}
\hline \multirow[t]{2}{*}{ Luz } & \multirow{2}{*}{$\begin{array}{l}\text { Moluscos ou girino } \\
\text { presente na câmara }\end{array}$} & \multicolumn{2}{|c|}{$\begin{array}{c}\text { Percentagem de miracídios atraídos para } \\
\text { a câmara } A\end{array}$} \\
\hline & & $\mathrm{BH}$ & SJ \\
\hline $\begin{array}{l}\text { Ausência } \\
\text { Presença } \\
\text { Ausência } \\
\text { Presença } \\
\text { Presença } \\
\text { Presença } \\
\text { Presença }\end{array}$ & $\begin{array}{l}\text { B. glabrata } \\
\text { B. glabrata } \\
\text { B. tenagophila } \\
\text { B. tenagophila } \\
\text { (*) B. glabrata } \\
\text { (*) B. tenagophila } \\
\text { Hy la fuscovaria }\end{array}$ & $\begin{array}{c}61,78 \pm 12,47(10) \\
72,42 \pm 10,75(30) \\
50,60 \pm 15,98(13) \\
59,89 \pm 12,10(30) \\
47,16 \pm 19,74(34) \\
- \\
52,07 \pm 13,66(35)\end{array}$ & $\begin{array}{r}59,79 \pm 12,98(25) \\
48,54 \pm 22,82(10) \\
72,48 \pm 12,95(20) \\
68,56 \pm 12,57(10) \\
- \\
39,61 \pm 19,48(27) \\
54,07 \pm 12,52(30)\end{array}$ \\
\hline
\end{tabular}

( ) Número de experimentos.

(*) A câmara B continha um espécime de $B$. tenagophila que atraiu $35,63 \pm 17,95 \%$ dos miracídios e $22,38 \pm 8,21 \%$ permaneceram no canal.

(*) A câmara B continha um espécime de B. glabrata que atraiu 42,46 $\pm 22,13 \%$ dos miracídios e 26,82 \pm $15,57 \%$ permaneceram no canal.

BH - Belo Horizonte, MG.

SJ - Sáo José dos Campos, SP.

mujos alopátricos, os miracídios atacavam e penetravam pelo tegumento sem antes cumprir um ritual de volteios em torno do molusco.

A atração dos miracídios pelo muco e fezes de caramujos alopátricos manifestou-se moderadamente.

Quando depositamos um girino, numa das câmaras, observamos que numerosos miracídios concentravam-se ao redor da larva do batráquio, alguns tentando penetrá-la.

Pareceu-nos que, devido ao constante movimento do girino, os miracídios năo conseguiam penetrar no seu tegumento.

\section{ANÅLISE ESTATISTICA DOS RESULTADOS}

a) Atraçāo de miractdios das linhagens $B H e$ $S J$ frente $a \mathrm{~B}$. glabrata $e a \mathrm{~B}$. tenagophila.

Apenas para efeito de facilidade de expressão e de tabelamento dos dados, designamos a câmara onde aleatoriamente foi depositado o molusco ou o girino de câmara
A e a que permaneceu somente com água de câmara $B$.

$O$ objetivo do experimento foi determinar se os miracídios apresentavam preferência pelo molusco simpátrico e, no caso dessa hipotese ser confirmada, quantificar o fenô. meno.

0 número de experimentos realizados para cada combinação dos fatores luminosidade, linhagem do miracídio e espécie do molusco pode ser verificado na Tabela 2 .

Considerando-se $\mathbf{P}$ como a proporção dos miracídios que se dirigiam a câmara $A$, foi feita a transformação $Y=$ arco seno $\sqrt{ } P$.

Foi ajustado um modelo fatorial com ter. mos para os efeitos principais de luz, miracídio e caramujo e suas respectivas interaçoes.

Uma extensa análise de resíduos foi realizada, nada tendo sido revelado que pudesse prejudicar a análise.

Os resultados da análise de variância constam das Tabelas 3 e 4.

As interações luz-miracídios e caramujos-miracídios foram altamente significativas (Tabela 5 e 6). 
BRASIO, B. de C. et al. Atraçăo de miracídios de Schistosoma mansoni por hospedeiros invertebrados. Comportamento de miracídios frente a girinos de Hyla fuscovaria. Rev. Saúde pribl., S. Paulo, 19:18$27,1985$.

T A B E L A 2

Número de variáveis utilizadas por experimento, segundo luminosidade, espécie do molusco e linhagem do miracídio.

\begin{tabular}{llll}
\hline Luminosidade & Espécie do molusco & BH & Miracídio \\
\hline Ausência & B. glebrata & 10 & 24 \\
de luz & B. tenagophila & 12 & 18 \\
presença & B. glabrata & 30 & 10 \\
de luz & B. tenagophila & 29 & 13 \\
\hline
\end{tabular}

BH - Belo Horizonte, MG.

SJ - Săo Jose dos Campos, SP.

T A B E L A 3

Resultado da análise de variância.

\begin{tabular}{lrcccc}
\hline Fonte & GL & SQ & QM & F & Prob. F \\
\hline modelo & 7 & 1,1196 & 0,1599 & 8.77 & 0,0001 \\
erro & 138 & 2,5172 & 0,0182 & & \\
Total & 145 & 3,6368 & & & \\
\hline
\end{tabular}

TAB E L A 4

Análise de variância detalhada.

\begin{tabular}{lccccc}
\hline Fonte & GL & SQ Tipo 1 & Prob. F & SQ Tipo VI & Prob. F \\
\hline Luz & 1 & 0,0110 & 0,4386 & 0,0000 & 0,9664 \\
Car & 1 & 0,0000 & 0,9489 & 0,0326 & 0,1834 \\
Luz $*$ Car & 1 & 0,1392 & 0,0065 & 0,0019 & 0,7503 \\
Mir & 1 & 0,0009 & 0,8215 & 0,0005 & 0,8745 \\
Luz $*$ Mir & 1 & 0,2595 & 0,0002 & 0,2994 & 0,0001 \\
Car * Mir & 1 & 0,6947 & 0,0001 & 0,6781 & 0,0001 \\
Luz $*$ Car * Mir & 1 & 0,0141 & 0,3800 & 0,0141 & 0,3800 \\
\hline
\end{tabular}

\$ Nota: As somas dos quadrados do tipo I referem-se ao efeito do termo correspondente, ajustado para os que $o$ antecedem, ignorando-se os que o seguem. As do tipo IV referem-se ao efeito do termo correspondente, ajustado para todos os demais no modelo.

A interação luz-caramujo está confundida com o conjunto dos outros fatores, devido à desigualdade dos números de observaçóes. b) Atração de miracidios das linhagens $B H e$ foi realizada em duas fases:
SJ frente a presenca concomitante de caramujos das espécies B. glabrata $e$ B. tenagophila.

$A$ análise estatística deste experimento 
BRASIO, B. de C. et al. Atração de miractdios de Schistosoma mansoni por hospedeiros invertebrados. Comportamento de miracidios frente a girinos de Hyla fuscovaria. Rev. Saide públ., S. Paulo, 19:1827, 1985.

\section{T A B E L A 5}

Médias por combinações de fatores luz-miracídios.

\begin{tabular}{lcc}
\hline & \multicolumn{2}{c}{ Miracídios } \\
\cline { 2 - 3 } Luz & BH & SJ \\
\hline $\begin{array}{c}\text { Ausência } \\
\text { (A) }\end{array}$ & $\begin{array}{c}0,8520 \pm 0,114 \\
(22)\end{array}$ & $\begin{array}{c}0,9470 \pm 0,0599 \\
(42)\end{array}$ \\
$\begin{array}{c}\text { Presença } \\
\text { (P) }\end{array}$ & $\begin{array}{c}0,9553 \pm 0,0427 \\
(59)\end{array}$ & $\begin{array}{c}0,8716 \pm 0,1094 \\
(23)\end{array}$ \\
\hline
\end{tabular}

Observação: Os valores entre parêntesis referem-se ao número de observaçōes de cada tipo de experimento. BH - Belo Horizonte, MG.

SJ - São José dos Campos, SP.

T AB E L A 6

Médias da interação caramujos-miracídios.

\begin{tabular}{|c|c|c|}
\hline \multirow[b]{2}{*}{ Caramujos } & \multicolumn{2}{|c|}{ Miracídios } \\
\hline & BH & SJ \\
\hline $\begin{array}{l}\text { B. glabrata } \\
\text { BG } \\
\text { B. tenagophile } \\
\text { BT }\end{array}$ & $\begin{array}{c}0,9951 \pm 0,629 \\
(40) \\
0,8610 \pm 0,0614 \\
(41)\end{array}$ & $\begin{array}{c}0,8414 \pm 0,0740 \\
(34) \\
1,0068 \pm 0,0812 \\
(31)\end{array}$ \\
\hline
\end{tabular}

BH - Belo Horizonte, MG.

SJ - Stao José dos Campos, SP.

\section{I) Estudo das variáveis}

YA: proporção de miracídios atraídos pelo molusco simpátrico.

YO: proporção de miracídios atraídos pelo molusco alopátrico.

Essas variáveis foram apenas consideradas para estudar o efeito de atração simpátrica. II) Estudo das variáveis:

PG: número de miracidios, classsificados por linhagem, atraídos por $B$. glabrata.

PT: número de miracídios, classificados por linhagem, atraidos por B. tenagophila.

Em ambos os casos, por serem as variáveis, proporçðes, adotamos a transformação: $Y=$ arco seno $\sqrt{ } P$ onde $0<P<1$ para sistematizar nossas observações.

Os dados foram verificados por diversos meios exploratórios, quanto à presença de valores aberrantes, simetria e distribuição empírica comparada à Gaussiana. Resíduos dos modelos lineares foram também estuda. dos desta forma.

A analise conjuntiva da YA $\mathrm{YO}$, através de análises de variância multivariada, quanto ao efeito diferencial do miracidio, forneceu o nível de significância $X=0,06$ pelos critérios de Wilks e de Pillai. Os testes semivariados forneceram resultados significativos para a variável $Y A$ com $X=0,02$. Esta foi a única diferença significativa. As proporções observadas constam da Tabela 7 .

Pelo que se observou no estudo da atração de miracidios das linhagens $\mathrm{BH}$ e $\mathrm{SJ}$ frente a $B$. glabrata e a $B$. tenagophila (item a da presente andilise estatística), resolvemos analisar o número de miracidios atraídos para $B$. glabrata e $B$. tenagophila já que no 
BRASIO, B. de C. et al. Atração de miracídios de Schistosome mansoni por hospedeiros invertebrados. Comportamento de miracídios frente a girinos de Hyle fuscoverla. Rev. Saúde públ., S. Paulo, 19:1827,1985 .

item anterior demos ênfase apenas a atração pelo caramujo simpátrico.

\section{T A B E L A ?}

Proporçōes observadas de miracídios atraídos pelo molusco simpátrico (YA).

\begin{tabular}{lcc}
\hline Miracídios & YA & N \\
\hline BH & 0,467 & 33 \\
SJ & 0,348 & 27 \\
\hline
\end{tabular}

BH - Belo Horizonte, MG.

SJ - São José dos Campos, SP.

Procedemos a duas análises de variância cujos resultados estão nas Tabelas 8 e 9 . Estas duas análises de variância são inequí vocas na determinação de que a linhagem do miracídio não influi no resultado.

A analise estatística dos resultados mostrou que na presença concomitante das duas espécies de caramujo, as linhagens de miracídios utilizadas não têm influência na atração. No entanto, $B$. glabrata mostrou maior atração que $B$. tenagophila, para ambas a li- nhagens de miracídios, nas condiçðes do presente experimento.

c) Atraç̄o de miracidios das linhagens $B H e$ $S J$ frente a girinos de Hyla fuscovaria.

0 objeto do experimento foi determinar se os miracídios apresentavam preferência pela câmara contendo o girino.

Os dasos obtidos no experimento estão sumarizados na Tabela 10 , de acordo com as preferências observadas $\mathrm{x}^{2}=3,26(2 \mathrm{gl})$; $\alpha>0,10$. Logo, as distribuições, pelas câmaras A e B e pelo canal, são as mesmas para os miracídios BH e SJ.

Analisando a atração temos: $\hat{\mathrm{p}}_{\mathrm{A}}=0, \overline{529}$; $\hat{\mathbf{p}}_{\mathbf{B}}=0,361 ; \hat{\mathrm{p}}_{\mathrm{C}}=0,110$.

Neste processamento, se o girino náo exercesse atração sobre os miracídios, deveríamos esperar que metade dos miracídios estivesse na câmara $A$ e a outra metade na câmara $B$ (desprezando o canal de pequeno volume).

Podemos ver que $\hat{\mathbf{p}}_{\mathrm{A}}$ diferiu pouco de 0,5 e um teste aproximado daria:

$Z=\underline{0,529-0,5} ; 663=1,49 \operatorname{com} \alpha>0,1$. $0,529 \times 0,471$

A análise mostrou que não houve evidên. cia de atração dos girinos sobre os miracídios.

T A B E L A 8

Analise de variância do comportamento de miracídios BH frente a B. tenagophlla

\begin{tabular}{lcccc}
\hline Fonte & GL & SQ & QM & "F" \\
\hline Mir & 1 & 0,0011 & 0,0011 & 0,02 \\
Erro & 57 & 2,7483 & 0,0482 & \\
Total (corr.) & 58 & 2,7494 & & \\
\hline
\end{tabular}

TA B E L A 9

Análise de variância do comportamento de miracídio SJ frente a B. glebrata

\begin{tabular}{lcccc}
\hline Fonte & GL & SQ & QM & "F" \\
\hline Mir & 1 & 0,0051 & 0,0051 & 0,09 \\
Erro & 58 & 3,3864 & 0,0584 & \\
Total (corr.) & 59 & 3,3915 & & \\
\hline
\end{tabular}


BRASIO, B. de C et al. Atração de miracídios de Schistosoma mansoni por hospedeiros invertebrados. Comportamento de miracidios frente a girinos de Hyla fuscovaria. Rev. Saride públ., S. Paulo, 19:1827, 1985 .

T A B E L A 10

Distribuição dos miracídios pelas câmaras.

\begin{tabular}{lccc}
\hline & \multicolumn{2}{c}{ Miracídios } & Total \\
\cline { 2 - 4 } Câmara & $\overline{B H}$ & SJ & 351 \\
\hline Câmara & 188 & 163 & \\
A & $(191,118)$ & $(159,882)$ & 239 \\
Câmara & 139 & 100 & \\
B & $(130,134)$ & $(108,866)$ & 73 \\
Canal & 34 & 39 & \\
Total & $(39,748)$ & $(33,252)$ & 663 \\
\hline $\mathbf{x}^{2}=3,26$ & 361 & 302 &
\end{tabular}

Observação: $\mathbf{A}$ câmara onde os girinos foram aleatoriamente depositados recebeu a designação de câmara $A$. BH - Belo Horizonte, MG.

SJ - Sŕo José dos Campos, SP.

\section{DISCUSSÃO E CONCLUSÕES}

$\mathrm{O}$ aparelho de duas câmaras mostrou-se plenamente satisfatório para nossos prop6sitos, pois permitiu visualização total do canal através da lupa estereoscópica, possibilitando a contagem dos miracídios que se dirigiam para cada uma das câmaras.

As análises estatísticas dos primeiros resultados mostraram que a luz influiu de maneira significativa nos experimentos. Devido a este fato, resolvemos fazer experimentos com e sem iluminação artificial e comparar, a seguir, os resultados.

Quando o aparelho de duas câmaras foi usado com um caramujo em cada câmara, ño foi possivel realizar estes experimentos com ausência de luz. Após colocar os miracídios no canal, aguardamos algum tempo e acendíamos as luzes. Como a maioria dos miracídios já havia penetrado pelo tegumen. to dos moluscos, não era possivel avaliar o número deles que havia se dirigido para cada câmara.

Ao colocar um girino de Hyla fuscovaria numa das câmaras observamos que, muitas vezes, quando os miracídios se aproximavam e tentavam penetrar no tegumento do girino, este se agitava vigorosamente, afugentan- do os miracídios para o canal. Por essas razões, esses experimentos tiveram que ser rea. lizados sob constante observação e, conseqüentemente, em presença de luz.

Após a análise estatística e em decorrência simplesmente de nossas observaçoes pudemos chegar a conclusões e em outros casos aventar hipóteses interessantes.

Os miracídios, quando em presença de uma só espécie de caramujo, apresentam maior atração para a espécie simpátrica, sugerindo-se aí a constatação de uma curiosa adaptação a nível de linhagem do tremató. deo e da espécie do planorbídeo.

Os miracídios $\mathrm{BH}$ apresentaram maior atração pelos caramujos na presença de luz e os miracídios $\mathrm{SJ}$ na ausência de luz. Se esta observação corresponder aos fatos ocorrentes nos focos naturais de doença podemos sugerir que os miracídios de $S$. mansoni da linhagem BH infectariam a $B$. glabrata preferentemente de dia e os miracídios da linhagem SJ infectariam $B$. tenagophila preferentemente à noite.

Quando da presença simultânea das duas espécies de caramujo, os miracídios não discriminam seu vetor; mesmo assim a espécie $B$. glabrata atraiu mais os miracídios das duas linhagens. E justificável aventar a hipótese de 
BRASIO, B. de C. et al. Atração de miraćádios de Schistosoma mansoni por hospedeiros invertebrados. Comportamento de miracídios frente a girinos de Hyla fuscovaria. Rev. Saúde públ., S. Paulo, 19:18$27,1985$.

que, em presença de substâncias quimiotáxicas provenientes das duas espécies de moluscos, os miracídios percam a capacidade de discriminá-las.

Os miracidios da linhagem $\mathrm{BH}$ parecem ser atraidos mais vigorosamente pelos moluscos simpátricos do que os miracídios $\mathrm{SJ}$ pelos moluscos simpátricos. Esta observação reforça outras observaç̃es de outras fases do ciclo biológico do verme que mostraram que a linhagem mineira além de estar mais adaptada ao vetor apresenta aspectos de maior vi- talidade no hospedeiro vertebrado. (Magalhães e Carvalho', 10 (1969, 1976); Magalhães e col. ${ }^{11}$ (1975).

Como, frente aos girinos de Hyla fuscovaria, os miracídios não foram atraídos, parece ser evidente que a presença de substâncias quimiotáxicas emanadas pelos vetores do $S$. mansoni (condição que facilita a manutenção do cilco vital do trematódeo), teve caráter adaptativo constituindo aspecto impor. tante a ser considerado na interação verme-molusco.

BRASIO, B. de C. et al. [Miracidia attraction to invertebrate hosts. Miracidia behavior in the presence of Hyla fuscovaria girina]. Rev. Saúde públ, S. Paulo, 19:18-27, 1985.

ABSTRACT: Miracidia tropism to suitable hosts is a subject of much controversy. In this work, we try to determine the possible specificity of Schistosoma mansoni miracidia attraction and also the degree of this specificity. To this end an experiment was carried out to determine the probability of attraction in a given experimental set up, to $H$. fuscovaria girina or to one of two susceptible snail species. Two strains of $S$. mansoni were employed and also the corresponding snail species. A specially designed apparatus consisting of two circular glass chambers joined by an open channel was used. One of the girina or a snail was placed in one of the chambers (randomly chosen). In the channel a known number of miracidia were deposited and the count of miracidia in each of the three compartments (the two chambers and the channel) was recorded. The experiment was carried out under strong or subdued lighting. Statistical and ad hoc exploratory data analysis have shown that: a) the miracidia, when exposed to only one species of snail, show more attraction to the sympatric snail host; b) one strain of miraciadia showed more attraction under strong lighting; the other strain behaved the opposite way, with stronger attraction under weak liht; c) the girina did not attract the miracidia; this seems to imply that sone chemiotropism is present in this phenomenon.

UNITERMS: Schistosoma mansoni Biomphalaria glabrata. Biomphalaria tenagophila. Hyla fuscovaria.

\section{REFERENCIAS BIBLIOGRÁFICAS}

1. BARBOSA, F.A.S. Alguns aspectos das relaçôes hospedeiro-parasita entre as fases larvárias do trematódeo Schistosome mansoni e o molusco Australorbis glabratus. Bol: Fund. Gonçalo Moniz, 15: 144, 1960.

2. BARBOSA, F.A.S. Ecology of the larval parasitic stages of Schistosoma mansoni. Rev. Inst. Med. trop. S. Paulo, 7:112-20, 1965.

3. BARBOSA, F.A.S. \& CARNEIRO, F. Penetration of Schistosoma mansoni miracidia in abnormal hosts. Rev. Inst. Med. trop. S. Paulo, 7: 99-102, 1965.

4. ETGES, F.S. \& DECKER, C.I. Chemosensitivity of the miracidium of Schistosome mansoni to Australorbis glabratus and other snail. J. Parasit., 49: 114-6, 1963.

5. KAWAZOE, U.; MAGALHAES, L.A.; MOTTA, L.K. \& TAKAKU, L. Competição biológica entre Biomphalaria glabrata (Say, 1818) e Biomphalaria tenagophila (D'Or- 
BRASIO, B. de C. et al. Atração de miracídios de Schistosoma mansoni por hospedeiros invertebrados. Comportamento de miracídios frente a girinos de Hyla fuscovaria. Rev. Saude príbl., S. Paulo, 19:18. 27,1985 .

bigny, 1835), em criadouros naturais, no município de Ourinhos, SP (Brasil). Rev. Saúde públ, S. Paulo, 14:65-87, 1980

6. KLOETZEL, K. Observaçð̃es sobre o tropismo do miracídio do Schistosoma mansoni pelo molusco Australorbis glabratus. Rev. bras. Biol., 18: 223-32, 1958.

7. KLOETZEL, K. Novas observações sobre o tropismo do miracídio de Schistosome mansoni pelo molusco Australorbis glabra. tus. Rev. Inst. Med. trop. S. Paulo, 2:3416, 1960 .

8. MAGALHĀES, L.A. Estudo de uma população de Biomphalaria glabrato (Say, 1818) recentemente introduzida no Estado da Guanabara. Folia clin. biol., 35: 102-20, 1966.

9. MAGALHÃES, L.A. \& CARVALHO, J.F. Determinação do número de cercárias provenientes de cepas diferentes de Schistosoma mansoni que conseguem penetrar, sob determinadas condiçōes de laboratório, em Mus musculus. Rev. Soc. bras. Med. trop., 3: 249-51, 1969.

10. MAGALHĀES, L.A. \& CARVALHO, J.F. Sobre o comportamento de duas linhagens de Schistosoma mansoni Sambon, 1907. Proposição para método de estudo quantitativo. Rev. Soc. bras. Med. trop., 10: 169-94, 1976.

11. MAGALHĀES, L.A.; ALĊANTARA, F.G. \& CARVALHO, J.F. Alguns dados referentes ao estudo parasitológico e anatomopatológico de duas linhagens de Schistosoma mansoni Sambon, 1907. Rev. Saúde públ, S. Paulo, 9: 1-5, 1975.
12. MANSON, P.R. \& FIPP, P.S. Chemical stimulation of Schistosoma mansont miracidial activity. Z. Parasitenk, 53: 287-95, 1977.

13. PARAENSE, W.L. \& CORREA, L.R. Sobre a ocorrência de duas raças biológicas de Schistosoma mansoni no Brasil Ciênc. Cult., 15: 245-6, 1963.

14. PARAENSE, W.L. \& CORREA, L.R. Susceptibility of Australorbis tenagophilus to infection with Schistosoma mansoni Rev. Inst. Med. trop. S. Paulo, 5: 23-9, 1963.

15. PARAENSE, W.L. \& CORREA, Variation in susceptibility of Australorbis glabratus to a strains of Schistosoma mansoni Rev. Inst. Med. trop. S. Paulo, 5: 15-22, 1963.

16. ROBERTS, T.M.; LINCK, R.W. \& CHERNIN, E. Effector mechanism of the response of Schistosome mansoni miracidia to snail conditioned water. J. exp. Zool, 211 13742,1980 .

17. UPATHAM, E.S. Expessure of caged Biom phalaria glabrata (Say) to investigate dispersion of miracidia of Schistosoma man soni Sambon in out door habitats in St. Lucia. J. Helminth., 46: 297-306, 1972.

18. WILSON, E.O. Chemical communication within animal species. In: Chemical ecology. New York, Academic Press, 1970. p. 133 . 55 .

Recebido para publicação em 24/07/1984 Reapresentado em 28/12/1984 Aprovado para publicaçāo em 07/01/1985 